Schlafapnoe

\title{
„Schnarchprothese“: Alternative mit positiver Langzeitwirkung
}

Saletu A et al. Langzeiteffekte der Unterkieferprotrusionsschiene IST ${ }^{\circledR}$

Somnologie 2010;14:41-47

\section{Hintergrund und Fragestellung}

Bei Patienten, die eine CPAP-Therapie ablehnen, und bei solchen mit sog. primärem Schnarchen sowie milder bis mäßiggradiger Schlafapnoe sind die Unterkieferprotrusionsschienen („Schnarchprothesen") eine anerkannte Alternative zur CPAP-Therapie. Bei richtiger Indikationsstellung ist der Akuteffekt der Unterkieferprotrusionsschienen belegt [2].

Ziel dieser Arbeit war die Erfassung der Langzeitwirkungen und -nebenwirkungen sowie der Patientencompliance bei der Anwendung der Unterkieferprotrusionsschiene IST $^{\circledR}$ nach Hinz.

\section{Methodik}

44 Patienten (11 Frauen, 33 Männer, 43-83 Jahre) im Schlaflabor mittels Polysomnografie auf die IST ${ }^{\circledR}$-Schiene eingestellte Patienten wurden nach einer mindestens zwei Jahre dauernden Therapie in die Studie aufgenommen. Bei sechs Patienten lag primäres Schnarchen vor, bei 38 eine obstruktive Schlafapnoe, davon fünf mit einer schwergradigen Schlafapnoe mit einem Apnoe-Hypopnoe-Index (AHI) von > 30/h Schlaf.

Die Studienteilnehmer beantworteten mehrere validierte schlafmedizinische Fragebögen, u.a. Pittsburgh-Schlafqualitätsindex (PSQI), Epworth-SleepinessScale (ESS) und andere Fragebögen zur Selbstbeurteilung sowie zur Erfassung etwaiger Nebenwirkungen.

\section{Ergebnisse}

Die Tragedauer der IST ${ }^{\circledR}$-Schiene lag zwischen 24 und 75 Monaten (Mittelwert: 32 Monate). $77 \%$ der antwortenden Patienten verwendeten die Schiene noch regelmäßig.

Die Therapieeffizienz zeigte sich u.a. in der Abnahme des von den Partnern gehörten Schnarchens von 93\% auf 26\% nach der zweijährigen Behandlung. Zu einem Therapieabbruch kam es bei zehn von 44 Patienten (23\%) wegen eines zu geringen Effekts, eines Umstiegs auf eine alternative Therapie oder eines unangenehmen bzw. schmerzhaften Empfindens während der Anwendung.

Der PSQI-Index nahm signifikant ab und erreichte fast Normwerte. Der ESSIndex war zwar vor der Behandlung im Mittel nur grenzwertig erhöht $(9,1)$ und nahm nach einem Monat signifikant $\mathrm{ab}$ $(6,9)$, war dann aber nach 24 Monaten nicht mehr signifikant verschieden vom Ausgangswert $(8,4)$.

Die Verträglichkeit der Schiene wurde von 69\% der Befragten als "sehr gut“ oder „gut“ eingeschätzt. Häufige Nebenwirkungen waren vermehrter Speichelfluss (59\%), Mundtrockenheit (51\%), Kiefergelenkschmerzen (35\%) und Zahnschmerzen (32\%). Kiefergelenkbeschwerden führten aber nur in $7 \%$ der Fälle zum Therapieabbruch.

\section{Schlussfolgerungen}

Die IST ${ }^{\circledR}$-Schiene ist auch im Langzeitgebrauch eine wirksame Behandlungsoption für primäres Schnarchen und Schlafapnoe. Regelmäßige schlafmedizinische wie auch zahnärztliche Kontrollen sind aber zur Vermeidung von Nebenwirkungen und zur Optimierung der Patientencompliance unerlässlich.

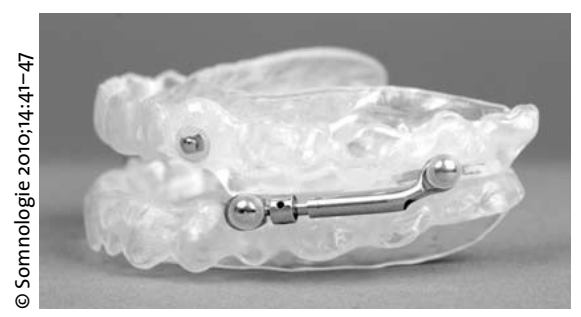

Schnarchtherapiegerät IST ${ }^{\circledR}$ : Die Führungsteleskope erlauben mandibuläre Öffnungs- und Seitwärtsbewegungen.

\section{Kommentar}

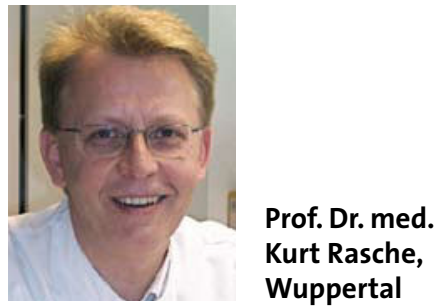

Die Effizienz der Therapie mit einer Unterkieferprotrusionsschiene scheint auch nach zweijähriger Anwendung gegeben zu sein. Auffällig ist allerdings, dass sich der ESSScore nicht signifikant verändert. Dies könnte an dem niedrigen, nahezu im Normbereich liegenden Ausgangswert vor der Therapie liegen. Grundlage hierfür ist, dass ohnehin in der Regel nur Patienten mit einer leicht- bis mittelgradig ausgeprägten Schlafapnoe auf eine Unterkieferprotrusionsschiene eingestellt werden (sollten). Eine Ausnahme stellen nur Patienten mit schwergradiger Schlafapnoe dar, die eine CPAP-Therapie nicht akzeptieren. Der Anteil dieser Patienten betrug in der Studie nur ca. $11 \%$.

Bei den anderen Fragebögen kam es dagegen zu einer anhaltenden signifikanten Besserung der abgefragten Parameter. Das spricht für die lang anhaltende Wirksamkeit der Therapie.

Der Anteil von Nebenwirkungen wie vermehrter Speichelfluss, Mundtrockenheit oder Kiefergelenksbeschwerden war mit z.T. über $50 \%$ erheblich, führte aber offensichtlich nur sehr selten zum Therapieabbruch. Die Therapietreue war mit 77\% dementsprechend, aber eigentlich unerwartet hoch. Die Therapietreue ist sicherlich in starkem Maße abhängig von regelmäßigen schlafmedizinischen und zahnärztlichen bzw. kieferorthopädischen Kontrollen. Eine Therapie mit Unterkieferprotrusionsschienen bei Schlafapnoe sollte daher - wie dies auch für die CPAP-Therapie analog gefordert wird - nur von einem interdisziplinären, schlafmedizinisch erfahrenen Expertenteam angepasst und überwacht werden, damit therapeutische Fehlentwicklungen vermieden werden können.

\section{Literatur}

1. Giles TL et al. Cochrane Database 2006; Syst Rev 3:CDoo1106

2. Hoffstein V. Sleep Breath 2007;11:1-22

3. Ghazal A et al. J Sleep Res. 2009;18:321-328 